\title{
Transcranial direct current stimulation for balance and gait in repetitive mild traumatic brain injury in rats
}

Gahee Park', Jee Hyun Suh ${ }^{1,2^{*}}$ and Soo Jeong $\operatorname{Han}^{1+}$

\begin{abstract}
Background: Balance impairment and lack of postural orientation are serious problems in patients with repetitive mild traumatic brain injury (mTBI).

Objective: To investigate whether anodal transcranial direct current stimulation (tDCS) over the primary motor cortex (M1) can improve balance control and gait in repetitive mTBI rat models.

Methods: In this prospective animal study, 65 repetitive mTBI rats were randomly assigned to two groups: the tDCS group and the control group. To create repetitive mTBI model rats, we induced mTBI in the rats for 3 consecutive days. The tDCS group received one session of anodal tDCS over the M1 area $24 \mathrm{~h}$ after the third induced $\mathrm{mTBl}$, while the control group did not receive tDCS treatment. Motor-evoked potential (MEP), foot-fault test, and rotarod test were evaluated before mTBI, before tDCS and after tDCS. The Mann-Whitney $U$ test and Wilcoxon signed rank test were used to assess the effects of variables between the two groups.

Results: Anodal tDCS over the M1 area significantly improved the amplitude of MEP in the tDCS group $(p=0.041)$. In addition, rotarod duration was significantly increased in the tDCS group $(p=0.001)$. The foot-fault ratio was slightly lower in the tDCS group, however, this was not statistically significant.

Conclusion: Anodal tDCS at the M1 area could significantly improve the amplitude of MEP and balance function in a repetitive $\mathrm{mTBl}$ rat model. We expect that anodal tDCS would have the potential to improve balance in patients with repetitive $\mathrm{mTBI}$.
\end{abstract}

Keywords: TDCS, Mild traumatic brain injury, Balance, Motor evoked potential

\section{Introduction}

Mild traumatic brain injury (mTBI), also known as concussion, is a head injury that temporarily affects brain function. They commonly occur in adolescents and young adults who practice sports and driving. Previous studies have shown that 144,000 children and adolescents

\footnotetext{
*Correspondence: suhtwins@hanmail.net

${ }^{\dagger}$ Jee Hyun Suh and Soo Jeong Han contributed equally to this work

${ }^{1}$ Department of Rehabilitation Medicine, College of Medicine,

Ewha Womans University, 1071 An-Yang-Cheon Ro, Yang-Cheon Gu,

Seoul 07985, Republic of Korea

Full list of author information is available at the end of the article
}

visit emergency departments for mTBI per year, [1] with full incidence of mTBI estimated to be as high as 3.8 million annually in both adolescents and adults [2]. The symptoms of mTBI include non-motor symptoms such as headache, loss of consciousness, and memory loss, but there are also motor symptoms such as balance impairment, lack of motor coordination and decreased dynamic motor function [3, 4]. Because of these symptoms, patients with mTBI have difficulty returning to exercise or daily life. In addition, when mTBI occurs repetitively, prognosis is poor. Parkinson's disease and chronic traumatic encephalopathy, which both affect balance and 
motor coordination, may occur after repetitive mTBI [5]. Repetitive mTBI is a source of significant economic burden in terms of days lost from work and costs related to medical treatment [4]. Recently, with the increasing practice of exercise and driving increase, the frequency of repetitive mTBI has also increased. However, there are few studies on the treatment of sequelae caused by repetitive mTBI. Furthermore, previous studies have focused primarily on non-motor defects rather than on motor deficits. It is important to further evaluate these motor deficits caused by repetitive mTBI as they may be a cause of long-term problems for patients.

Transcranial direct current stimulation (tDCS) is a form of neuromodulation that uses a constant, low direct current delivered via electrodes to the head. tDCS is a non-invasive, easy to handle, and low-cost technique that induces regional changes in neuronal excitability [6]. Previous studies have reported that $\mathrm{tDCS}$ has a therapeutic effect in patients with neurological disorders, such as Parkinson's disease, Alzheimer's disease, and stroke [79]. tDCS has emerged as a promising therapeutic tool to improve balance and postural control [6]. In a previous study, tDCS applied at the primary motor cortex (M1) in individuals with cerebral palsy and healthy young adults resulted in improved balance [6]. However, no study has assessed the effect of anodal tDCS on balance and postural orientation in patients with repetitive mTBI.

The brains of humans and rats are anatomically similar, which makes rats good models for studying human brain disease [10]. In humans, mTBI varies with rotational force, acceleration-deceleration, and degree of impact $[11,12]$. Therefore, it is difficult to fit the baseline similarly. Therefore, in this study, we want to verify the effectiveness of anodal tDCS at the M1 area by making rat model to check the baseline function and making a uniform rat model with repetitive mTBI. The purpose of this study was to investigate the changes in electrophysiology, balance control, and postural orientation as an effect of anodal tDCS at the M1 area in rat models with repetitive mTBI. We hypothesized that anodal tDCS at the M1 area would improve balance impairment after repetitive mTBI.

\section{Material and methods}

This prospective, randomized animal study was approved by the Institutional Animal Care and Use Committee of Ewha medical research institute (approval number 13-0235). Sixty-five healthy male Sprague-Dawley rats (Orient Bio, Seongnam, Korea) weighing 220-280 g supplied by a single-source breeder were used in this study. Based on a previous literature, six-week-old rats were used in this study as this age corresponds to the late juvenile to early adulthood stage in rats, which is the common age of mTBI occurrence in humans [13]. Hormonal levels influence the cortical excitability and neurotransmitter levels which affect the tDCS response [14-16]. And the male would receive more current at the cortex than female due to the cortical bone structure [17]. Furthermore, males make up larger percentage of cases than females in mTBI [18]. For these reasons, in this study, only male rats were included. The animals were housed in a rodent facility at $23.0 \pm 1.0^{\circ} \mathrm{C}$ with 12 -h light-dark cycle, and had free access to tap water and regular rat chow. All animals received human care in compliance with the National Institutes of Health guidelines for the use of experimental animals. This study was carried out in compliance with the ARRIVE guidelines.

\section{Experimental design}

The treatment time and test schedule are shown in Fig. 1a. Each of the 65 rats used were randomized to the tDCS $(n=33)$ and control groups $(n=32)$. The tDCS group received one session of anodal tDCS treatment at $24 \mathrm{~h}$ after repetitive mTBI (day 4), while the control group did not receive tDCS treatment after repetitive mTBI.

\section{Animal preparation}

All procedures and evaluations, except motor coordination studies, were performed under anesthesia. Anesthesia was initiated with an intramuscular injection of tiletamine/zolazepam (15 mg/kg; Zoletil ${ }^{\circledR}$, Vibac, France).

The induction of repetitive mTBI was conducted for 3 consecutive days (days 1-3) in all rats (Fig. 1). mTBI was induced in rats using a modified weight-drop device and a protocol previously described by Tang et al. [19]. A $175 \mathrm{~g}$ steel weight was briefly dropped from a height of $30 \mathrm{~cm}$ through a polyvinyl chloride tube with an inner diameter of $11 \mathrm{~mm}$ terminating on the bregma of the rat. The rat was placed on a wooden plate and fixed with Velcro in the prone position. To evaluate the possibility of a skull fracture or brain hemorrhage, we conducted brain MRI study on all rats after completing the study on day 5 . The rats were anesthetized with Zoletil ${ }^{\circledR}$ and placed in a $5 \mathrm{~cm}$ inner diameter, 4-element phased-array, animaldedicated surface coil (Chenguang Medical Technology, Co., Ltd, Shanghai, China). The strength of the MRI magnet was $3 \mathrm{~T}$. The MRI protocol used was a T2-weighted image sequence (repetition time/echo time $=650 / 22$ ). The slice thickness was $3.0 \mathrm{~mm}$ and the matrix scan size was $512 \times 512$ pixels. Brain MRI showed no significant pathological features, such as skull fracture, brain hemorrhage, or diffuse axonal injury after repetitive mTBI (Fig. 2a, b). The histological examination was performed to rule out the presence of brain damage resulting from repetitive mTBI at day 5 . Brains of rats were removed and fixed in $10 \%$ neutral buffered formalin after being 
a

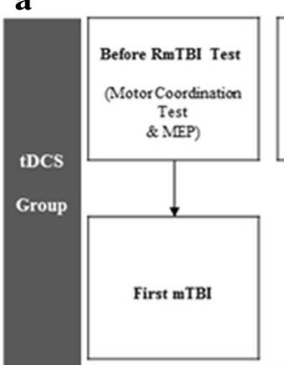

Day 1
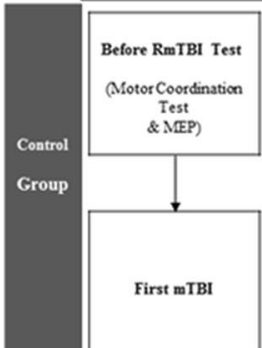
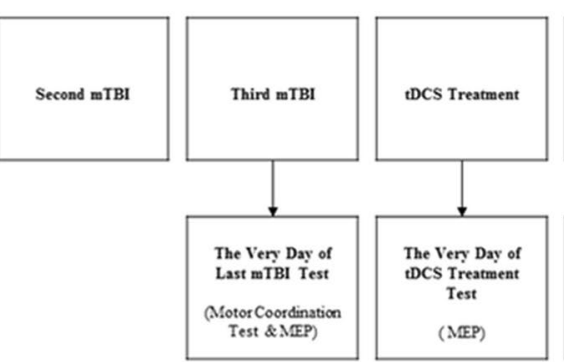

Day 3

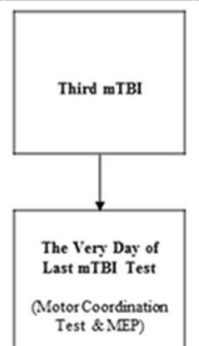

Day 4

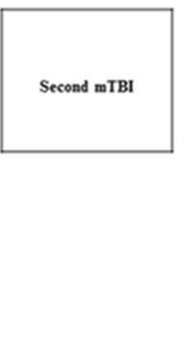

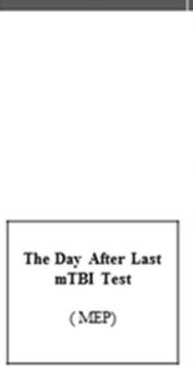

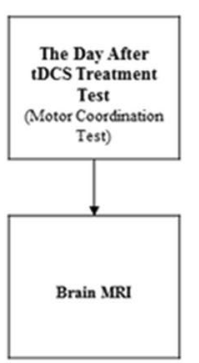

Day 5

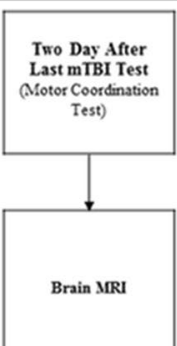

b

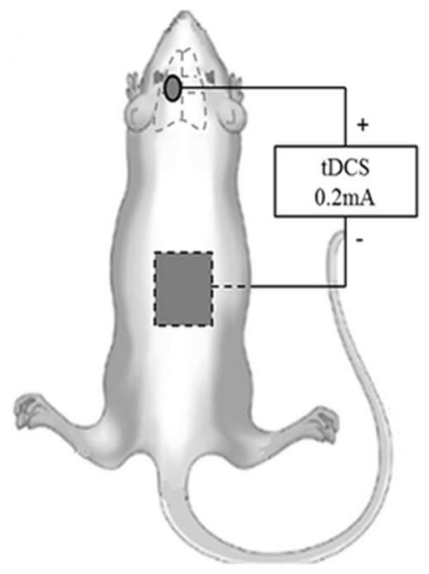

Fig. 1 Experimental arrangement and the position of electrodes during tDCS. a Experimental arrangement. Before induction of repetitive mTBI (day 1), MEP and behavioral tests are performed on the rat model. After pre-mTBI tests, the first mTBI is induced in the rat models. On days 2 and 3 , second and third $\mathrm{mTBI}$ is induced in the rats to establish the repetitive $\mathrm{MTBI}$ rat models. After the third mTBI, MEP, and motor coordination studies are done (day 3). On day 4, anodal tDCS at M1 area is applied to the tDCS group. On day 4, $1 \mathrm{~h}$ after tDCS, MEP is done. On day 5, behavioral tests and brain MRI are done. $\mathbf{b}$ The position of anodal and cathodal electrodes during anodal tDCS. Circle means anodal electrode and square means cathodal electrode. Cup-shaped anodal electrode was attached to the skin over the left M1 area, and rectangular rubber cathodal electrode was positioned on the trunk

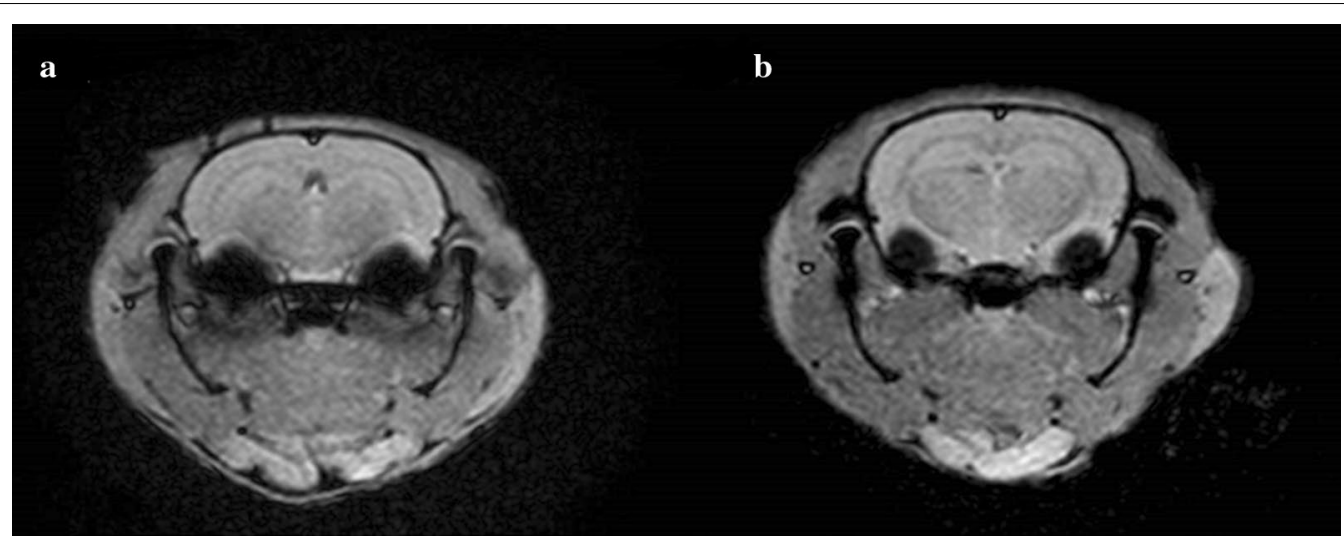

Fig. 2 Brain magnetic resonance imaging (MRI) of repetitive mTBI rats. a Brain MRI of control group, b Brain MRI of tDCS group. Brain MRI showed no significant pathological features after repetitive $\mathrm{MTBI}$ in either the control or tDCS groups

euthanized in a closed chamber with $10 \%$ carbon dioxide. Coronal brain Sects. $15 \mu \mathrm{m}$ thick were cut using a microtome, mounted on glass slides, and stained with hematoxylin and eosin. Light microscopy was used for evaluating morphological changes in brain tissue at $100 \times$ magnification. Histochemical analysis showed no visible morphological changes in either the control or tDCS groups (Fig. 3a, b).

\section{Experimental procedures}

After three inductions of mTBI, the rats in the tDCS group received a single session of anodal tDCS under anesthesia, $24 \mathrm{~h}$ after the last induction of mTBI (day 4, Fig. 1a), while those in the control group received only anesthesia without anodal tDCS stimulation. The fur around the bregma was removed to ensure tight attachment of the anodal electrode. An $10 \mathrm{~mm}$ diameter 

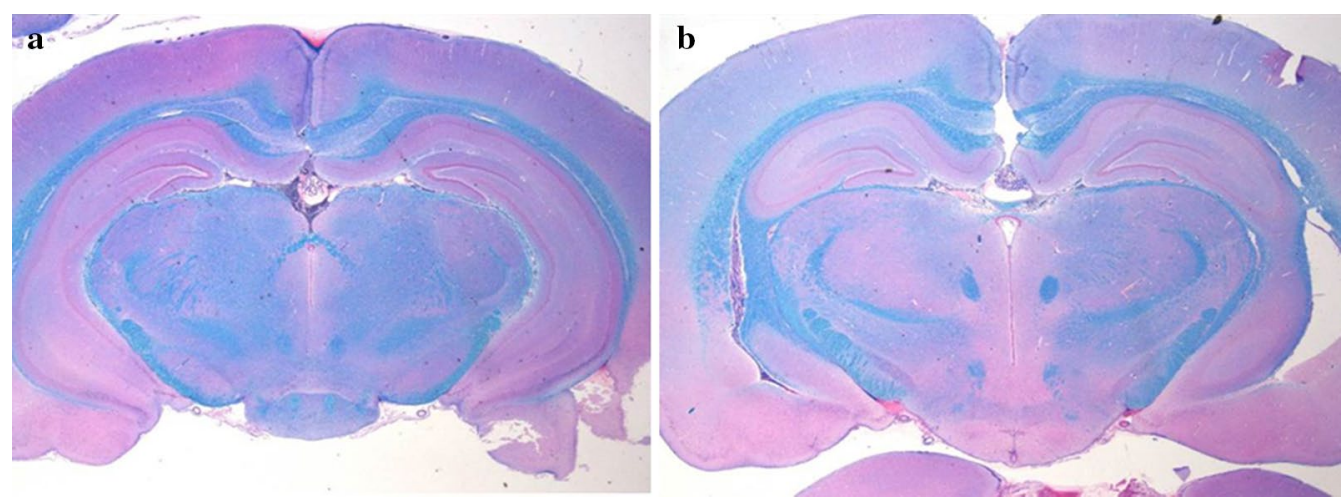

Fig. 3 Hematoxylin and eosin staining in repetitive mTBI rats. a Brain MRI of control group, b Brain MRI of tDCS group. Histochemical analysis showed no morphological changes in either the control or tDCS groups

$\left(0.785 \mathrm{~cm}^{2}\right.$ contact area), cup-shaped anodal electrode was attached to the skin over the left M1 area, $3 \mathrm{~mm}$ to the left and $2 \mathrm{~mm}$ in front of the interaural line (Fig. 1b) [20]. The $30 \times 30 \mathrm{~mm}^{2}$ rectangular rubber cathodal electrode was positioned on the trunk and wrapped with a bandage (Fig. 1b) [21, 22]. The salt-free, chloride free electrically conductive gel was filled in cup-shaped anodal electrode and applied to the rubber cathodal electrode. A constant direct current was applied via stimulator (PhoresorII ${ }^{\circledR}$, IOMED, Salt Lake City, UT, USA), with an intensity of $0.2 \mathrm{~mA}$ and a current density of $0.255 \mathrm{~mA} /$ $\mathrm{cm}^{2}$ for $30 \mathrm{~min}[21,23,24]$. tDCS was performed by a single experienced physiatrist.

\section{Measurements}

To evaluate the functional integrity of the motor system, transcranial motor-evoked potentials (MEPs) were evaluated. MEPs are muscle action potentials elicited by transcranial magnetic brain stimulation [25]. In this study, MEP measurements were evaluated pre-mTBI (day 1 ), immediately post-mTBI (day 3 ), and $1 \mathrm{~h}$ posttDCS (day 4) to evaluate the excitability of the corticospinal pathway. MEPs at the bilateral tibialis anterior muscles of the hind limbs were evaluated. The right MEP was recorded from the tibialis anterior muscle of the right hindlimb, which resulted from left motor cortex stimulation (Fig. 4a). The left MEP, which was used as the control variable, was recorded from the tibialis anterior muscle of the left hindlimb, which resulted from the right motor cortex stimulation (Fig. 4b). The active needle electrode was inserted into the belly of the tibialis anterior muscle, and the reference needle electrode was inserted into the distal part of the tibialis anterior muscle. The ground electrode was placed on an opposite footpad. MEPs were recorded using a Medtronic Keypoint ${ }^{\circledR}$ (Medtronic Inc., Jacksonville,

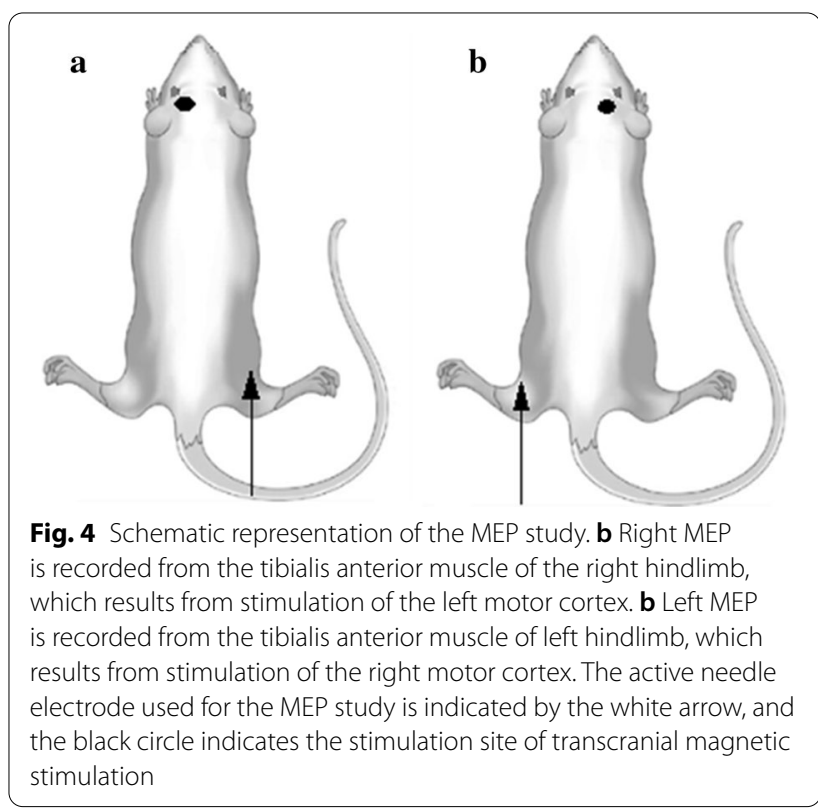

FL, USA) at a sweep velocity of $5 \mathrm{~ms}$ with a sensitivity of $200 \mu \mathrm{V}$. The band-pass filter was set at $20-10 \mathrm{kHz}$. Single-pulse transcranial magnetic stimulation was administered using a magnetic stimulator Magstim ${ }^{\circledR}$ (Magstim Company, Whiteland, Wales, UK) and a figure-eight magnetic coil (diameter per loop $=50 \mathrm{~mm}$, peak magnetic field $=4.0 \mathrm{~T}$ ). The center of the coil was positioned on the motor cortex, whose center was anterior and lateral to the bregma on the contralateral side of the hindlimb, where the active needle electrode was inserted (Fig. 4a,b). A total of 20 MEPs were recorded at $10 \mathrm{~s}$ inter-stimulus intervals [26]. TMS intensity was recorded as percent machine output(MO), with $100 \%$ corresponding to the maximal amplitude electrical current conducted through the magnetic coil. We set the 
stimulation intensity to $100 \% \mathrm{MO}$ [24]. The intensity of the stimulation was maintained constant throughout the procedure. The average latency of three representative waves was calculated with reference to previous research, [26] and the highest value was analyzed for peak-to-peak amplitude in the mean of three waves; latency was defined as the interval before the initial deflection.

To evaluate balance control and postural orientation, the foot-fault test [27] and the rotarod test were conducted. The foot-fault test has been found to objectively demonstrate impairments in motor coordination and sensorimotor function, and rehabilitation effects after ischemia in rodents [28]. The rotarod test was used to assess motor coordination and balance alterations in rodents [28]. These tests were conducted pre-mTBI (day 1 ), on the day of the last mTBI (day 3 ), and 2 days postmTBI (day 5,1 day post-tDCS), to eliminate anesthetic effects. In the foot-fault test, an elevated $52 \times 40 \mathrm{~cm}^{2}$ stainless steel metal grid with grid cells of $3 \times 3 \mathrm{~cm}^{2}$ was used. The rats were placed in the center of a metal grid and observed for one min via video recording. A foot fault was considered when the hind limbs fell between the grid cells or when the paw was correctly placed on the grid but slipped during weight bearing for hind limbs. ${ }^{29}$ The foot-fault ratio was obtained by dividing the number of foot faults by the total number of footsteps on hind limbs [29]. A rotarod treadmill was used to conduct the rotarod test. The rotation speed was set at $15 \mathrm{rpm}$, and a rat was placed at the center of a $9 \mathrm{~cm}$ diameter metal roller. The trial lasted up to $3 \mathrm{~min}$, and the time during which the rat was able to stay on the roller was recorded [30]. Three trials were performed, and the average value was calculated.

To eliminate bias, MEPs and balance and postural orientation tests were performed by an experienced physiatrist blinded to the group allocations.

\section{Statistical analysis}

When the Kolmogorov-Smirnov test and Shapiro-Wilk test were performed to use the parametric method, the normality was not satisfied. The Mann-Whitney U test was used to compare the values of electrophysiological measurements and balance and postural orientation tests between the tDCS and control groups. The Wilcoxon signed rank test was used to compare the values of electrophysiological measurements between day 3 and day 4 , and to compare the values of the balance and postural orientation tests between day 3 and day 5 in the tDCS and control groups, respectively. Statistical analysis was performed using SPSS version 21.0 (IBM SPSS, Armonk, NY, USA), and p-values less than 0.05 , were considered statistically significant.

\section{Results \\ MEPs}

The amplitudes and latencies of MEP on days 1, 3, and 4 showed no significant difference between the tDCS group and the control group. The amplitude of the right MEP recorded from the tibialis anterior muscle of the right hindlimb, which resulted from left motor cortex stimulation, progressively declined from day 1 to 3 in both groups. The amplitude of right MEP was significantly different between day 3 and day 4 in the tDCS group $(0.069 \pm 0.042$ versus $0.158 \pm 0.223 \mathrm{mV}, \mathrm{p}=0.041)$ (Table 1; Fig. 5a), whereas there was no significant difference between day 3 and day 4 in the control group. There was no significant difference in the amplitudes of the right MEP between the tDCS group and the control group measured on day 4 (Fig. 5a). In addition, no significant difference was observed in the bilateral MEP latencies recorded at the bilateral tibialis anterior muscles between days 3 and 4 (Fig. 5c, d).

In this study, since tDCS was applied to the left M1 area, the measurement of the change in the right MEP was of interest, and the left MEP was used as a control for the MEP test. There was no significant difference

Table 1 The results of motor-evoked potential (MEP) evaluation performed in each group

\begin{tabular}{|c|c|c|c|c|c|c|}
\hline \multirow[t]{2}{*}{ Evaluation } & \multicolumn{3}{|l|}{ tDCS group } & \multicolumn{3}{|l|}{ Control group } \\
\hline & Day 1 & Day 3 & Day 4 & Day 1 & Day 3 & Day 4 \\
\hline Right MEP amplitude (mV) & $0.117 \pm 0.230$ & $0.069 \pm 0.042$ & $0.158 \pm 0.223^{*}$ & $0.072 \pm 0.059$ & $0.060 \pm 0.051$ & $0.070 \pm 0.045$ \\
\hline Left MEP ${ }^{\dagger \dagger}$ amplitude (mV) & $0.045 \pm 0.049$ & $0.079 \pm 0.067$ & $0.187 \pm 0.533$ & $0.046 \pm 0.070$ & $0.063 \pm 0.043$ & $0.072 \pm 0.072$ \\
\hline Right MEP' latency (ms) & $4.471 \pm 1.830$ & $4.813 \pm 0.721$ & $5.451 \pm 1.850$ & $4.313 \pm 2.055$ & $4.873 \pm 0.800$ & $4.815 \pm 0.821$ \\
\hline Left MEP ${ }^{\dagger \dagger}$ latency (ms) & $5.022 \pm 0.870$ & $5.137 \pm 0.825$ & $5.497 \pm 1.745$ & $4.751 \pm 1.088$ & $5.026 \pm 0.923$ & $4.832 \pm 0.904$ \\
\hline
\end{tabular}

Values are mean \pm standard deviation

+ Right MEP, MEP recorded at right hind limb

${ }^{+\dagger}$ Left MEP, MEP recorded at the left hind limb

${ }^{*} p<0.05$ 


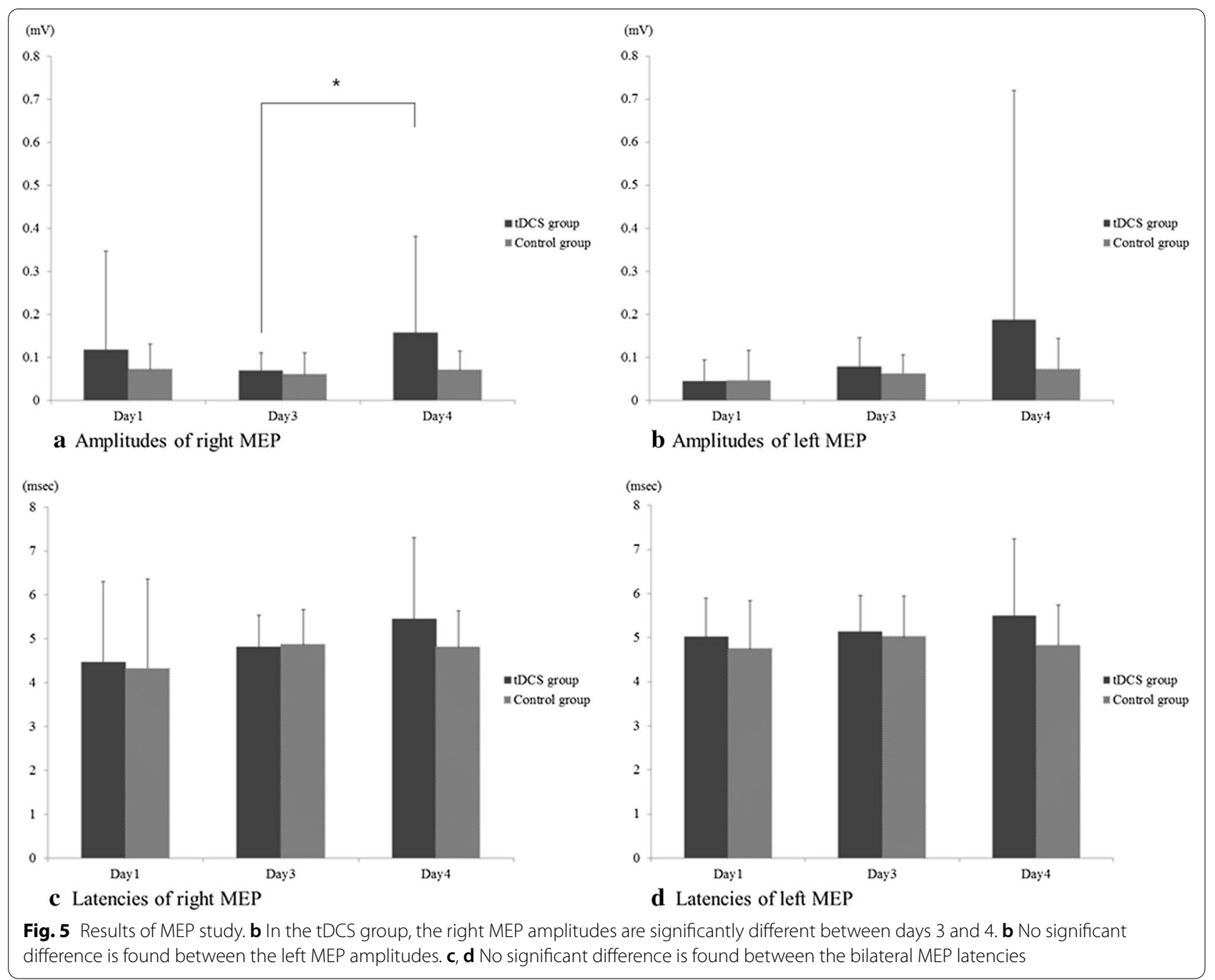

in the amplitudes of the left MEP recorded at the left tibialis anterior muscle between days 3 and 4 in each group (Fig. 5b). There was no significant difference in the amplitudes of the left MEP between the tDCS and control groups measured on day 4 (Fig. 5b).

\section{Balance and postural orientation tests}

Although not statistically significant, the foot fault ratio showed declining trend when comparing day 3 and day 5 in the tDCS group (Table 2; Fig. 6a).

Based on the results of the foot-fault test, the foot-fault ratio in the tDCS group was slightly lower than that of

Table 2 The results of motor coordination studies performed in each group

\begin{tabular}{|c|c|c|c|c|c|c|}
\hline \multirow[t]{2}{*}{ Evaluation } & \multicolumn{3}{|l|}{ tDCS group } & \multicolumn{3}{|l|}{ Control group } \\
\hline & $\begin{array}{l}\text { Day } 1 \\
\text { (Pre- repetitive mTBI) }\end{array}$ & $\begin{array}{l}\text { Day } 3 \\
\text { (Pre-tDCS) }\end{array}$ & $\begin{array}{l}\text { Day } 5 \\
\text { (Post-tDCS) }\end{array}$ & $\begin{array}{l}\text { Day } 1 \\
\text { (Pre- repetitive mTBI) }\end{array}$ & $\begin{array}{l}\text { Day } 3 \\
\text { (Pre-tDCS) }\end{array}$ & $\begin{array}{l}\text { Day } 5 \\
\text { (Post-tDCS) }\end{array}$ \\
\hline Foot-fault ratio & $0041 \pm 0.028$ & $0.033 \pm 0.062$ & $0.015 \pm 0.029$ & $0.032 \pm 0.035$ & $0.018 \pm 0.034$ & $0.019 \pm 0.038$ \\
\hline Rota rod duration (sec) & $40.561 \pm 47.346$ & $66.621 \pm 68.797$ & $103.697 \pm 76.470^{*}$ & $33.995 \pm 44.437$ & $95.422 \pm 76.166$ & $91.141 \pm 76.146$ \\
\hline
\end{tabular}

Values are mean \pm standard deviation

$m T B I$ mild traumatic brain injury, $t D C S$ transcranial direct current stimulation

${ }^{*} \mathrm{p}<0.05$ 


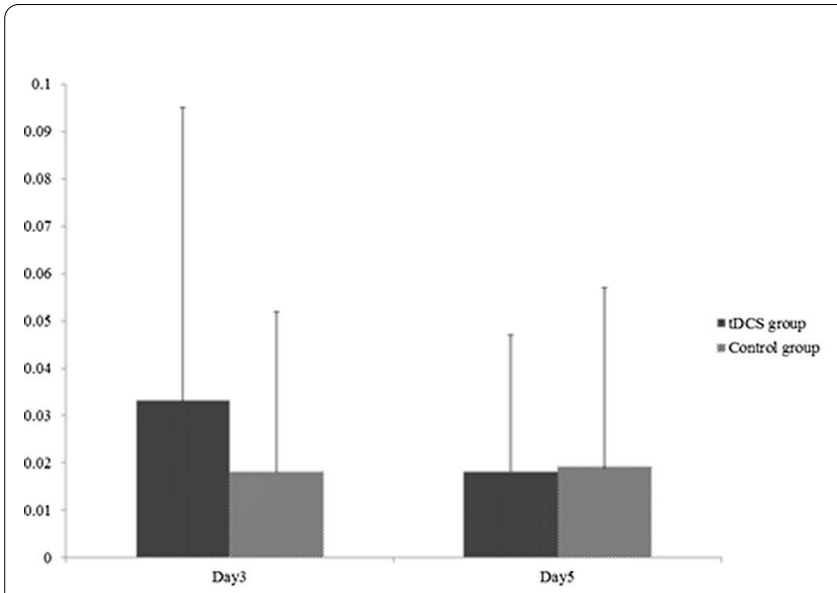

a Foot-fault ratio

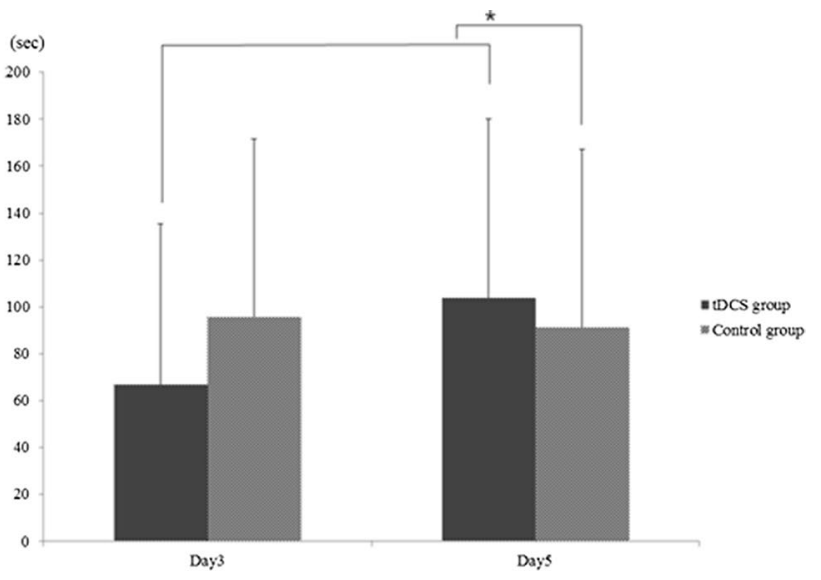

b Rota rod duration

Fig. 6 Results of behavioral studies. a The foot-fault ratio is slightly lower in the tDCS group than in the control group, however, the results are not statistically significant. $\mathbf{b}$ The rotarod duration on day 5 is significantly increased as compared with that on day 3 in the tDCS group. c The rotarod durations on day 5 are significantly different between the tDCS group and the control group

the control group, however, this difference was not statistically significant (Table 2; Fig. 6a).

The rotarod duration was significantly increased on day 5 compared with day 3 in the tDCS group $(66.621 \pm 68.797 \mathrm{~s}$ versus $103.697 \pm 76.470 \mathrm{~s}, \mathrm{p}=0.001)$ (Fig. 6b). There was a significant difference between the rotarod durations of the tDCS group and the control group measured on day $5(103.697 \pm 76.470$ versus $91.141 \pm 76.146 \mathrm{~s}, \mathrm{p}=0.006$ ).

In the control group, there was no statistically significant difference in the results of the foot-fault test and rotarod test between days 3 and 5 .

\section{Discussion}

$\mathrm{n}$ this study, the influence of anodal tDCS at the M1 area on amplitude of MEP and balance function after repetitive mTBI was evaluated. The results demonstrated that anodal tDCS at the M1 area, which underwent immediately after repetitive $\mathrm{mTBI}$, increased the amplitude of MEP, showed a decreasing trend of the foot-fault ratio, and improved the rotarod duration. tDCS may have therapeutic benefits for balance function and electrophysiological changes. To the best of our knowledge, this study is the first to suggest an increase in corticospinal excitability and balance improvement after anodal tDCS on the M1 area in a large number of repetitive mTBI rat models by analyzing MEP studies and behavioral tests.

Standing and gait are critical for most activities of daily living. In patients with mTBI, balance instability and gait alteration are predictors of increased fall risk, loss of functional independence, morbidity, and mortality. A previous study showed that mTBI patients had a greater sway area, larger mediolateral displacement amplitude, and slower body oscillation than healthy individuals [31]. Martini et al. showed that mTBI patients adopted a more conservative gait strategy and slower gait than those without a history of mTBI $[32,33]$. To maintain balance, the central nervous system must effectively integrate sensory and motor information through complex mechanisms involving cortical and subcortical pathways [34]. Previous studies suggest that combinations of central and peripheral deficits may be the cause of balance and gait alterations in mTBI $[35,36]$. Balance and gait alteration occurs through the following two mechanisms: (1) the brain centers responsible for central integration of vestibular, visual, and somatosensory information may be impaired, or (2) the peripheral receptors themselves may be damaged and provide inaccurate senses of motion. A recent study showed that a higher incidence of asymmetry of the corticospinal tract (CST) was found in patients with mTBI [37]. The injury to the CST leads to problems in balance and coordination [37]. Furthermore, Karayannidou et al's study provided insight into how pyramidal tract neurons (PTNs) from the fore- and hindlimb projections in the primary motor cortex respond to postural changes during two distinct tasks [38]. PTNs are the main output neurons of the motor cortex and influence the activity of motor neurons and interneurons in the ventral horn of the spinal cord. In other words, it may be assumed that anodal tDCS on the M1 area would have improved balance by activating the CST as well as the PTNs.

tDCS is a promising strategy to modulate brain network function and, in doing so, the supraspinal control of balance. tDCS safely and selectively modulates the excitability of brain networks [39]. tDCS targeting the primary 
sensorimotor regions has been demonstrated to improve balance in older adults and patients with stroke [40-42]. The M1 area is composed of the primary motor cortex and premotor cortex [43]. The CST originates from several cortical areas, and approximately half of these axons extend from neurons in the primary motor cortex. The primary function of the CST is voluntary motor control of the body and limbs. In this study, the amplitudes of the right MEP progressively declined from day 1 to 3 in both groups. This result demonstrated the disruption of the CST by repeated mTBI. This would have resulted in balance and gait alterations in the repetitive mTBI rat model. Anodal tDCS to the M1 area of repetitive mTBI rat models generates an electric field that polarizes neuronal populations and modulates resting membrane potentials. This activates the CST and induces balance and gait improvements.

In this study, a progressive decrease in the amplitude of right MEP after repetitive mTBI compared with before $\mathrm{mTBI}$, and a significant increase in the amplitude of right MEP after tDCS treatment compared with before tDCS treatment, was observed. The decreased MEP amplitude is evidence of decreased cortical excitability following repetitive $\mathrm{mTBI}$, and the increased MEP amplitude is evidence of increased corticospinal excitability following anodal tDCS [44]. MEP amplitude represents the total number of depolarized axons and innervated muscle fibers, and their degree of excitability [44]. Therefore, our results suggest that the number of motor units involved in corticospinal excitability was reduced by repetitive mTBI and could be restored by anodal tDCS in the M1 area. Replacement of lost fibers by the central nervous system may be facilitated by simultaneous excitement of motor units [45]. In contrast, no significant change was observed in the MEP latency of either group. The MEP latency reflects the speed at which signals arrive through the fastest-conducting nerve fibers. This suggests a mechanism in which anodal tDCS increases the number of axons involved in excitation but does not increase conduction velocity. These results are consistent with our previous study; the amplitude of MEP decreased after repetitive mTBI and increased after tDCS, but MEP latency was not affected [20].

In a previous tDCS study of the repetitive mTBI rat model, the sensory-evoked potential and MEP were measured to confirm transient motor recovery [20]. A previous study did not focus on balance, motor coordination, or gait impairment, and no behavioral tests were performed [20]. In addition, a previous study showed that a single session of anodal tDCS had little effect on balance improvement and postural control [46]. However, in this study, it was found that single-session tDCS helped in the activation of the CST and improvement of balance.
Another study suggested that multi-session anodal tDCS has an effect on balance improvement in children with cerebral palsy $[47,48]$. In order to further confirm the results of this study, it would be necessary to conduct comparative studies on multi-session anodal tDCS and single-session anodal $\mathrm{tDCS}$ in a repetitive mTBI rat model in the future.

Balance and posture are controlled by numerous interacting networks, such as the spinal cord, cerebellum, cortex, and brainstem $[49,50]$. Yosephi et al.s have suggested that bilateral stimulation of the cerebellar hemispheres is more effective than M1 area stimulation when anodal tDCS is implemented for the purpose of improving balance in older adults with high risk of falls [51]. However, a previous study showed that mTBI is associated with white matter and gray matter volume reduction and cortical thinning in areas including the M1 area, but does not affect the cerebellum [52]. Therefore, in this study, to confirm the effect of tDCS on balance improvement, the M1 area was stimulated rather than the cerebellum.

The rat is a key model for basic and preclinical studies of neuroscience, underlining its importance in studies of human disease. There are the close evolutionary and genomic relationship to humans, the sophistication and sociability of the animal, and the ease of physiological and behavioral measurements. Anodal tDCS on the M1 for balance and gait in rat model could be useful to further explore insight and may serve as a translational platform bridging human and animal studies, establishing new therapeutic strategies for repetitive mTBI.

\section{Limitation}

The present study has several limitations. First, a single session of anodal tDCS may be insufficient to significantly improve motor coordination and electrophysiology. Multiple sessions of anodal tDCS may produce clearer results than the current results; therefore, further research with multiple sessions of anodal tDCS in a repetitive $\mathrm{mTBI}$ rat model is needed. Second, in the present study, brain MRI was conducted 4 days after the first induction of mTBI and 2 days after the last induction of mTBI. It is known that repetitive mTBI can cause structural changes in the brain, such as cortical thinning and ventriculomegaly. In this study, brain MRI was conducted to evaluate the possibility that the weight drop device produced a skull fracture or brain hemorrhage. If brain MRI was performed after a longer period of time, changes in brain structure due to repetitive mTBI could have been confirmed in addition to ruling out skull fracture or hemorrhage. Third, as the rates of mTBI are higher in females than in males when similar sports are compared, anodal tDCS study on female are also needed [18]. In future study, the study 
on the effect of anodal tDCS at the M1 area in repetitive mTBI considering female's menstrual cycle would be needed. Fourth, immunochemical analysis was not conducted in this study. In subsequent studies, it would be necessary to apply some immunohistological analysis; antibody against BDNF to investigate effects on neural plasticity, GFAB to confirm the reactive astrocytosis and vGlut1 and GAD 65-67 markers to assess glutamate and GABA levels $[24,53,54]$. And in this study, the number of anesthesia was minimized to reduce side effects caused by anesthesia $[55,56]$. For this reason, MEP after tDCS was conducted on day 4 and motor coordination studies were performed on day 5 . There is sufficient time interval for neural plasticity to occur, and it makes some difficulty on the correlation between MEP and changes in behavioral performance. Since the MEP and motor coordination studies were examined on different days, it is difficult to prove that the results of the motor coordination study were not due to motor learning but due to an increase in corticospinal excitability. In the follow-up study, the correlation between the two tests would be increased by reducing the temporal interval between the two tests.

\section{Conclusions}

Balance impairments can increase the risk of falling, which may lead to the loss of functional independence and severe injuries. Therefore, improvement of balance and motor coordination function is an important treatment consideration in patients with repetitive mTBI to reduce the risk of falls and its consequences. This study proposes that anodal tDCS at the M1 area after repetitive $\mathrm{mTBI}$ could improve the amplitude of MEP as well as improve balance control, postural orientation and motor endurance by activating the CST. These results indicate that anodal tDCS can produce rapid, consistent, and controllable electrophysiological changes in corticomotor excitability in repetitive mTBI rat models. This newly developed tDCS protocol in a repetitive mTBI rat model may serve as a translational platform bridging human and animal studies, establishing new therapeutic strategies for patients with repetitive mTBI.

\footnotetext{
Abbreviations

mTBI: Mild traumatic brain injury; M1: Primary motor cortex; tDCS: Transcranial direct current stimulation; MEP: Motor-evoked potential; MO: Machine output; CST: Corticospinal tract; PTN: Pyramidal tract neuron.

\section{Acknowledgements}

This study was supported and funded by Basic Science Research Program through the National Research Foundation of Korea (NRF) funded by the Ministry of Education (NRF-2013R1A1A2059711). The funders had no role in study design, data collection and analysis, decision to publish, or preparation of the manuscript.
}

Authors' contributions

JHS and SJH projected this study and reviewed the manuscript. GP and JHS participated in the statistical analysis. GP interpretated the result and wrote the first draft. All authors read and approved the final manuscript.

\section{Availability of data and materials}

Data will be available upon reasonable request to the corresponding author.

\section{Declarations}

\section{Ethics Approval and Consent to Participate}

This study was approved the Institutional Animal Care and Use Committee of Ewha medical resarch institute (approval number 13-0235).

\section{Patient consent for publication}

Not required.

\section{Competing interests}

None declared.

\section{Author details}

1 Department of Rehabilitation Medicine, College of Medicine, Ewha Womans University, 1071 An-Yang-Cheon Ro, Yang-Cheon Gu, Seoul 07985, Republic of Korea. ${ }^{2}$ Department of Rehabilitation Medicine, Bundang Jesaeng General Hospital, 20, Seohyeon-ro 180 beon-gil, Bundang-gu, Seongnam-si, Gyeonggi-do 13590, Republic of Korea.

Received: 16 February 2021 Accepted: 6 April 2021

Published online: 17 April 2021

\section{References}

1. Meehan WP 3rd, Mannix R. Pediatric concussions in United States emergency departments in the years 2002 to 2006. J Pediatr. 2010;157(6):88993. https://doi.org/10.1016/j.jpeds.2010.06.040.

2. Schatz $P$, Moser RS. Current issues in pediatric sports concussion. Clin Neuropsychol. 2011;25(6):1042-57. https://doi.org/10.1080/13854046. 2011.556669.

3. Parker TM, Osternig LR, van Donkelaar P, Chou LS. Recovery of cognitive and dynamic motor function following concussion. Br J Sports Med. 2007;41(12):868-73. https://doi.org/10.1136/bjsm.2006.033761 (discussion 873).

4. Kushner D. Mild traumatic brain injury: toward understanding manifestations and treatment. Arch Intern Med. 1998;158(15):1617-24. https://doi. org/10.1001/archinte.158.15.1617.

5. Vanltallie TB. Traumatic brain injury (TBI) in collision sports: Possible mechanisms of transformation into chronic traumatic encephalopathy (CTE). Metabolism. 2019;100S:153943. https://doi.org/10.1016/j.metabol. 2019.07.007.

6. de Moura M, Hazime FA, Marotti Aparicio LV, Grecco LAC, Brunoni AR, Hasue RH. Effects of transcranial direct current stimulation (tDCS) on balance improvement: a systematic review and meta-analysis. Somatosens Mot Res. 2019;36(2):122-35. https://doi.org/10.1080/08990220.2019. 1624517

7. Fregni F, Boggio PS, Santos MC, et al. Noninvasive cortical stimulation with transcranial direct current stimulation in Parkinson's disease. Mov Disord. 2006;21(10):1693-702. https://doi.org/10.1002/mds.21012.

8. Hummel F, Celnik P, Giraux P, et al. Effects of non-invasive cortical stimulation on skilled motor function in chronic stroke. Brain. 2005;128(Pt 3):490-9. https://doi.org/10.1093/brain/awh369.

9. Ferrucci R, Mameli F, Guidi I, et al. Transcranial direct current stimulation improves recognition memory in Alzheimer disease. Neurology. 2008;71(7):493-8. https://doi.org/10.1212/01.wnl.0000317060.43722.a3.

10. Ellenbroek B, Youn J. Rodent models in neuroscience research: is it a rat race? Dis Model Mech. 2016;9(10):1079-87. https://doi.org/10.1242/dmm. 026120.

11. Bodnar CN, Roberts KN, Higgins EK, Bachstetter AD. A Systematic review of closed head injury models of mild traumatic brain injury in mice and 
rats. J Neurotrauma. 2019;36(11):1683-706. https://doi.org/10.1089/neu. 2018.6127.

12. Barth JT, Freeman JR, Broshek DK, Varney RN. Acceleration-deceleration sport-related concussion: the gravity of it all. J Athl Train. 2001;36(3):253-6

13. Holm L, Cassidy JD, Carroll LJ, Borg J. Neurotrauma task force on mild traumatic brain injury of the WHOCC. Summary of the WHO collaborating centre for neurotrauma task force on mild traumatic brain injury. J Rehabil Med. 2005:37(3):137-41. https://doi.org/10.1080/16501970510027321.

14. Smith MJ, Keel JC, Greenberg BD, et al. Menstrual cycle effects on cortical excitability. Neurology. 1999;53(9):2069-72. https://doi.org/10.1212/wnl. 53.9.2069.

15. Epperson CN, Haga K, Mason GF, et al. Cortical gamma-aminobutyric acid levels across the menstrual cycle in healthy women and those with premenstrual dysphoric disorder: a proton magnetic resonance spectroscopy study. Arch Gen Psychiatry. 2002;59(9):851-8. https://doi.org/10. 1001/archpsyc.59.9.851.

16. Batra NA, Seres-Mailo J, Hanstock C, et al. Proton magnetic resonance spectroscopy measurement of brain glutamate levels in premenstrual dysphoric disorder. Biol Psychiatry. 2008;63(12):1178-84. https://doi.org/ 10.1016/j.biopsych.2007.10.007.

17. Russell M, Goodman T, Wang Q, Groshong B, Lyeth BG. Gender differences in current received during transcranial electrical stimulation. Front Psychiatry. 2014;5:104. https://doi.org/10.3389/fpsyt.2014.00104.

18. Laker SR. Epidemiology of concussion and mild traumatic brain injury. PM R. 2011;3(10 Suppl 2):S354-8. https://doi.org/10.1016/j.pmrj.2011.07.017.

19. Tang YP, Noda Y, Hasegawa T, Nabeshima T. A concussive-like brain injury model in mice (I): impairment in learning and memory. J Neurotrauma. 1997;14(11):851-62. https://doi.org/10.1089/neu.1997.14.851.

20. Kim HJ, Han SJ. A simple rat model of mild traumatic brain injury: a device to reproduce anatomical and neurological changes of mild traumatic brain injury. PeerJ. 2017;5:e2818. https://doi.org/10.7717/peerj.2818.

21. Kim SJ, Kim BK, Ko YJ, Bang MS, Kim MH, Han TR. Functional and histologic changes after repeated transcranial direct current stimulation in rat stroke model. J Korean Med Sci. 2010;25(10):1499-505. https://doi.org/10. 3346/jkms.2010.25.10.1499.

22. Yoon KJ, Lee YT, Chae SW, Park CR, Kim DY. Effects of anodal transcranial direct current stimulation (tDCS) on behavioral and spatial memory during the early stage of traumatic brain injury in the rats. J Neurol Sci. 2016;362:314-20. https://doi.org/10.1016/j.jns.2016.02.005.

23. Lang N, Siebner HR, Ward NS, et al. How does transcranial DC stimulation of the primary motor cortex alter regional neuronal activity in the human brain? Eur J Neurosci. 2005;22(2):495-504. https://doi.org/10.1111/j.14609568.2005.04233.x.

24. Kim HJ, Han SJ. Anodal transcranial direct current stimulation provokes neuroplasticity in repetitive mild traumatic brain injury in rats. Neural Plast. 2017;2017:1372946. https://doi.org/10.1155/2017/1372946.

25. Grunhaus L, Polak D, Amiaz R, Dannon PN. Motor-evoked potential amplitudes elicited by transcranial magnetic stimulation do not differentiate between patients and normal controls. Int J Neuropsychopharmacol. 2003:6(4):371-8. https://doi.org/10.1017/S1461145703003705.

26. Luft AR, Kaelin-Lang A, Hauser TK, Cohen LG, Thakor NV, Hanley DF. Transcranial magnetic stimulation in the rat. Exp Brain Res. 2001:140(1):11221. https://doi.org/10.1007/s002210100805.

27. Rogers DC, Campbell CA, Stretton JL, Mackay KB. Correlation between motor impairment and infarct volume after permanent and transient middle cerebral artery occlusion in the rat. Stroke. 1997;28(10):2060-5. https://doi.org/10.1161/01.str.28.10.2060 (discussion 2066).

28. Schaar KL, Brenneman MM, Savitz SI. Functional assessments in the rodent stroke model. Exp Transl Stroke Med. 2010;2(1):13. https://doi.org/ 10.1186/2040-7378-2-13.

29. Horiquini Barbosa E, Vallim JH, Lachat JJ, de Castro VL. Assessments of motor abnormalities on the grid-walking and foot-fault tests from undernutrition in wistar rats. J Mot Behav. 2016;48(1):5-12. https://doi.org/10. 1080/00222895.2015.1024824.

30. Tiwari HS, Tripathi AK, Mishra DP, Kalita J, Misra UK. A study of ER stress in rat model of cerebral venous sinus thrombosis. Neurosci Lett. 2015:589:121-5. https://doi.org/10.1016/..neulet.2015.01.038.

31. Degani AM, Santos MM, Leonard CT, et al. The effects of mild traumatic brain injury on postural control. Brain Inj. 2017;31(1):49-56. https://doi. org/10.1080/02699052.2016.1225982.
32. Martini DN, Sabin MJ, DePesa SA, et al. The chronic effects of concussion on gait. Arch Phys Med Rehabil. 2011;92(4):585-9. https://doi.org/10. 1016/j.apmr.2010.11.029.

33. Wood TA, Hsieh KL, An R, Ballard RA, Sosnoff JJ. Balance and gait alterations observed more than 2 weeks after concussion: a systematic review and meta-analysis. Am J Phys Med Rehabil. 2019;98(7):566-76. https:// doi.org/10.1097/PHM.0000000000001152.

34. Powers KC, Kalmar JM, Cinelli ME. Dynamic stability and steering control following a sport-induced concussion. Gait Posture. 2014;39(2):728-32. https://doi.org/10.1016/j.gaitpost.2013.10.005.

35. Guskiewicz KM, Ross SE, Marshall SW. Postural stability and neuropsychological deficits after concussion in collegiate athletes. J Athl Train. 2001:36(3):263-73.

36. Mallinson Al, Longridge NS. Dizziness from whiplash and head injury: differences between whiplash and head injury. Am J Otol. 1998;19(6):814-8.

37. Maruta J, Mallott JM, Sulioti G, Ghajar J, Palacios EM, Mukherjee P. Concussion disrupts normal brain white matter microstructural symmetry. Front Neurol. 2020;11:548220. https://doi.org/10.3389/fneur.2020.548220.

38. Karayannidou A, Beloozerova IN, Zelenin PV, et al. Activity of pyramidal tract neurons in the cat during standing and walking on an inclined plane. J Physiol. 2009;587(Pt 15):3795-811. https://doi.org/10.1113/jphys iol.2009.170183.

39. Nitsche MA, Paulus W. Excitability changes induced in the human motor cortex by weak transcranial direct current stimulation. J Physiol. 2000;527(Pt 3):633-9. https://doi.org/10.1111/j.1469-7793.2000. t01-1-00633.x.

40. Nomura T, Kirimoto H. Anodal transcranial direct current stimulation over the supplementary motor area improves anticipatory postural adjustments in older adults. Front Hum Neurosci. 2018;12:317. https://doi.org/ 10.3389/fnhum.2018.00317.

41. Kaski D, Dominguez RO, Allum JH, Bronstein AM. Improving gait and balance in patients with leukoaraiosis using transcranial direct current stimulation and physical training: an exploratory study. Neurorehabil Neural Repair. 2013;27(9):864-71. https://doi.org/10.1177/1545968313 496328.

42. Saeys W, Vereeck L, Lafosse C, Truijen S, Wuyts FL, Van De Heyning P. Transcranial direct current stimulation in the recovery of postural control after stroke: a pilot study. Disabil Rehabil. 2015;37(20):1857-63. https://doi.org/ 10.3109/09638288.2014.982834.

43. Woolsey CN, Settlage PH, Meyer DR, Sencer W, Pinto Hamuy T, Travis AM. Patterns of localization in precentral and "supplementary" motor areas and their relation to the concept of a premotor area. Res Publ Assoc Res Nerv Ment Dis. 1952;30:238-64.

44. Cambiaghi M, Velikova S, Gonzalez-Rosa JJ, Cursi M, Comi G, Leocani L. Brain transcranial direct current stimulation modulates motor excitability in mice. Eur J Neurosci. 2010;31(4):704-9. https://doi.org/10.1111/j.14609568.2010.07092.x

45. Ikoma K, Samii A, Mercuri B, Wassermann EM, Hallett M. Abnormal cortical motor excitability in dystonia. Neurology. 1996;46(5):1371-6. https://doi. org/10.1212/wnl.46.5.1371.

46. Steiner KM, Enders A, Thier W, et al. Cerebellar tDCS does not improve learning in a complex whole body dynamic balance task in young healthy subjects. PLoS ONE. 2016;11(9):e0163598. https://doi.org/10. 1371/journal.pone.0163598.

47. Grecco LA, Oliveira CS, Duarte NA, Lima VL, Zanon N, Fregni F. Cerebellar transcranial direct current stimulation in children with ataxic cerebral palsy: a sham-controlled, crossover, pilot study. Dev Neurorehabil. 2017;20(3):142-8. https://doi.org/10.3109/17518423.2016.1139639.

48. Grecco LA, Duarte NA, Zanon N, Galli M, Fregni F, Oliveira CS. Effect of a single session of transcranial direct-current stimulation on balance and spatiotemporal gait variables in children with cerebral palsy: a randomized sham-controlled study. Braz J Phys Ther. 2014;18(5):419-27. https://doi.org/10.1590/bjpt-rbf.2014.0053.

49. Deliagina TG, Zelenin PV, Beloozerova IN, Orlovsky GN. Nervous mechanisms controlling body posture. Physiol Behav. 2007;92(1-2):148-54. https://doi.org/10.1016/j.physbeh.2007.05.023.

50. Jacobs JV, Horak FB. Cortical control of postural responses. J Neural Transm (Vienna). 2007;114(10):1339-48. https://doi.org/10.1007/ s00702-007-0657-0.

51. Yosephi MH, Ehsani F, Zoghi M, Jaberzadeh S. Multi-session anodal tDCS enhances the effects of postural training on balance and postural 
stability in older adults with high fall risk: primary motor cortex versus cerebellar stimulation. Brain Stimul. 2018;11(6):1239-50. https://doi.org/ 10.1016/j.brs.2018.07.044.

52. Sussman D, da Costa L, Chakravarty MM, Pang EW, Taylor MJ, Dunkley BT. Concussion induces focal and widespread neuromorphological changes. Neurosci Lett. 2017;650:52-9. https://doi.org/10.1016/j.neulet.2017.04. 026.

53. Du X, Li J, Li M, et al. Research progress on the role of type I vesicular glutamate transporter (VGLUT1) in nervous system diseases. Cell Biosci. 2020;10:26. https://doi.org/10.1186/s13578-020-00393-4.

54. Barde YA. Trophic factors and neuronal survival. Neuron. 1989;2(6):152534. https://doi.org/10.1016/0896-6273(89)90040-8.
55. Brammer DW, Doerning BJ, Chrisp CE, Rush HG. Anesthetic and nephrotoxic effects of Telazol in New Zealand white rabbits. Lab Anim Sci. 1991;41(5):432-5.

56. Saha DC, Saha AC, Malik G, Astiz ME, Rackow EC. Comparison of cardiovascular effects of tiletamine-zolazepam, pentobarbital, and ketaminexylazine in male rats. J Am Assoc Lab Anim Sci. 2007;46(2):74-80.

\section{Publisher's Note}

Springer Nature remains neutral with regard to jurisdictional claims in published maps and institutional affiliations.
Ready to submit your research? Choose BMC and benefit from:

- fast, convenient online submission

- thorough peer review by experienced researchers in your field

- rapid publication on acceptance

- support for research data, including large and complex data types

- gold Open Access which fosters wider collaboration and increased citations

- maximum visibility for your research: over $100 \mathrm{M}$ website views per year

At BMC, research is always in progress.

Learn more biomedcentral.com/submissions 